\title{
UCRL-CONF-217069
}

LAWRENCE LIVERMORE N A T IO N A L LABORATORY

\section{Imaging Performance of the Si/Ge Hybrid Compton Imager}

M. Burks, D. Chivers, C. Cork, M. Cunningham, L. Fabris, D. Gunter, E. Hull, D. Lange, H. Manini, L. Mihailescu, K. Nelson, T. Niedermayr, J. Valentine, K. Vetter, D. Wright

November 14, 2005

2005 IEEE Nuclear Science Symposium and Medical Imaging Conference

Fajardo, PR, United States

October 23, 2005 through October 29, 2005 
This document was prepared as an account of work sponsored by an agency of the United States Government. Neither the United States Government nor the University of California nor any of their employees, makes any warranty, express or implied, or assumes any legal liability or responsibility for the accuracy, completeness, or usefulness of any information, apparatus, product, or process disclosed, or represents that its use would not infringe privately owned rights. Reference herein to any specific commercial product, process, or service by trade name, trademark, manufacturer, or otherwise, does not necessarily constitute or imply its endorsement, recommendation, or favoring by the United States Government or the University of California. The views and opinions of authors expressed herein do not necessarily state or reflect those of the United States Government or the University of California, and shall not be used for advertising or product endorsement purposes. 


\title{
Imaging Performance of the Si/Ge Hybrid Compton Imager
}

\author{
Morgan Burks, Dan Chivers, Chris Cork, Mark Cunningham, Lorenzo Fabris, Donald Gunter, Ethan Hull, David \\ Lange, Hugh Manini, Lucian Mihailescu, Karl Nelson, Thomas Niedermayr, \\ John Valentine, Kai Vetter, Doug Wright
}

\begin{abstract}
The point spread function (PSF) of a fullyinstrumented silicon/germanium Compton telescope has been measured as a function of energy and angle. Overall, the resolution ranged from $3^{\circ}$ to $4^{0}$ FWHM over most of the energy range and field of view. The various contributions to the resolution have been quantified. These contributions include the energy uncertainty and position uncertainty of the detector; source energy; Doppler broadening; and the $1 / \mathrm{r}$ broadening characteristic of Compton back-projection. Furthermore, a distortion of the PSF is observed for sources imaged off-axis from the detector. These contributions are discussed and compared to theory and simulations.
\end{abstract}

\section{INTRODUCTION}

$\mathrm{T}$ he concept of using a Compton scatter telescope to image gamma-rays has been understood for several decades. However, detector and electronic limitations have prevented this technique from realizing its full potential [1]. Attempts at building Compton imagers resulted in instruments with very low efficiency. Furthermore, the limited energy resolution and/or lack of position granularity in the detectors made it difficult to achieve good imaging resolution. Recent advances in technology, especially the advent of segmented semiconductor detectors with fine position granularity and excellent energy resolution, have sparked interest in a new generation of Compton telescopes. These instruments promise a significantly higher efficiency, better energy resolution and better imaging resolution.

We have developed a fully-instrumented Compton telescope using two segmented planar detectors: a silicon strip detector and a germanium strip detector. The silicon strip detector has 32 channels on each side while the germanium has 38 channels on each side. The pitch of each detector is 2 $\mathrm{mm}$. The detectors are parallel, aligned on their center axis and separated by $6 \mathrm{~cm}$. This instrument is a prototype for a larger system that will eventually contain multiple silicon and germanium detectors. The reason for using the two types of semiconductor material is to allow for efficient Compton imaging ranging from $0.15 \mathrm{MeV}$ to $2 \mathrm{MeV}$. The instrument details are described separately [2].

\footnotetext{
Manuscript received November 10, 2005. This work was funded by the Office of Research and Development, Department of Homeland Security and performed under the auspices of the U.S. Department of Energy by University of California Lawrence Livermore National Laboratory under contract No. W7405-Eng-48.

All authors are with the Lawrence Livermore National Laboratory. Contact Morgan Burks (burks5@1lnl.gov).
}

The aim of the present work is to understand the resolution achievable by such a system and the various factors contributing to the point spread function (PSF). These factors include energy uncertainty, position uncertainty, Doppler broadening, the $1 / \mathrm{r}$ inherent PSF due to the Compton ring imaging, as well as the energy and location of the source. A combination of measurements and simulations were performed to study the imaging resolution.

\section{POINT SPREAD FUNCTION}

The sensitivity of a Compton imager depends on the efficiency and angular resolution of the device. This work focuses on the resolution and the various factors that broaden the resolution from the ideal response.

Energy resolution: The energy resolution of this system is nominally $2 \mathrm{keV}$ per channel at $662 \mathrm{keV}$. However, the resolution for a Compton event is $\sim \operatorname{sqrt}(3)$ higher because the event consists three interactions (on average), each of which is measured separately and contributes noise. The finite energy resolution affects the PSF because the scatter angle depends on the correct knowledge of the energy. However, it tends to be a weaker dependence than position resolution, especially at higher energies.

Position resolution: In our instrument, the lateral $\mathrm{x}$ and $\mathrm{y}$ positions are determined by the pitch size of $2 \mathrm{~mm}$. The depth of interactions is derived by the timing of the opposite electrodes. We have demonstrated a depth resolution of about $0.5 \mathrm{~mm}$ at $122 \mathrm{keV}$ and above. However, the $\mathrm{z}$ position is based on an analog measurement and can give poor results for interactions much below $60 \mathrm{keV}$.

Doppler Broadening: The angular resolution one can obtain in a Compton imager is ultimately limited by the Doppler broadening which is based on the intrinsic momentum of the electron involved in the Compton scatter process. The Doppler broadening depends on the $\mathrm{Z}$ of the detector material, the gamma-ray energy and the scattering angle. It is particularly important for small energies and larger scattering angles. The use of the low-Z Si detector in our instrument not only provides the necessary Compton scattering necessary for Compton imaging down below $150 \mathrm{keV}$ but also reduces the impact of the Doppler broadening over other materials such as Ge or CdZnTe.

1/R Compton "ring effect": If an ideal Compton imager with perfect performance could be constructed, it would still have a finite point response that would have the approximate form of $\mathrm{A} /(\mathrm{r}+1)$, where $\mathrm{A}$ is the amplitude and $\mathrm{r}$ is the radial 
distance. This form arises because each event reconstructs to a ring on the celestial sphere, giving a density of rings emanating from the source location. The purpose of image reconstruction is to remove this contribution in a manner analogous to tomographic reconstruction in PET.

Geometrical Bias: The geometry and finite size of the detector have a significant influence on the PSF. Quantifying this effect is important for understanding the efficiency, field of view, energy range and image reconstruction. This is discussed in detail below.

Data cuts: As with other imaging modalities, there is a tradeoff between efficiency and resolution. The quality of events vary considerably from one to the next with the primary distinction being the separation between subsequent interactions. Careful selection of events can result in an order of magnitude better resolution; however, many events must be discarded. Thus, for maximum sensitivity, the right tradeoff between efficiency and resolution must be found.

\section{Monte CARlo StUdy}

In order to examine the effects of position and energy uncertainty on the detector resolution as well as the effect of Doppler broadening, a Monte Carlo simulation was performed using GEANT 4. The simulation included the basic physics as well as the so-called Doppler broadening of the Compton interaction. The silicon and germanium detectors were modeled; however, in order to isolate the desired contributions, no dead materials (cryostat, PC boards etc.) were included in the model.

\section{MEASUREMENTS}

A series of measurements were made with a ${ }^{152}$ Eu point source in order to characterize the PSF as a function of position and energy. ${ }^{152} \mathrm{Eu}$ was chosen because it has a broad range of gamma-ray lines from $122 \mathrm{keV}$ to $1408 \mathrm{keV}$ (Fig. 1). The source was placed in the lab at distance of $50 \mathrm{~cm}$ from the detector and moved about the detector, in the horizontal plane, in $15^{\circ}$ increments. The data acquisition was triggered to only accept events that were coincident between the silicon and germanium detectors. This choice was made to affect a tradeoff between resolution and efficiency.

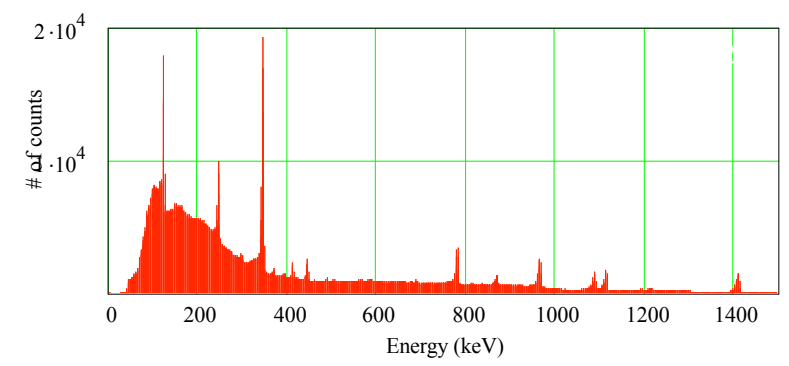

Fig. 1. Energy spectrum resulting from a ${ }^{152} \mathrm{Eu}$ source on the $\mathrm{Si} / \mathrm{Ge}$ Compton imager. The energy resolution is below $2 \mathrm{keV}$ (per channel) but multiple interactions per event combine to give a total resolution of $\sim 3.5 \mathrm{keV}$ (at 344 $\mathrm{keV})$.
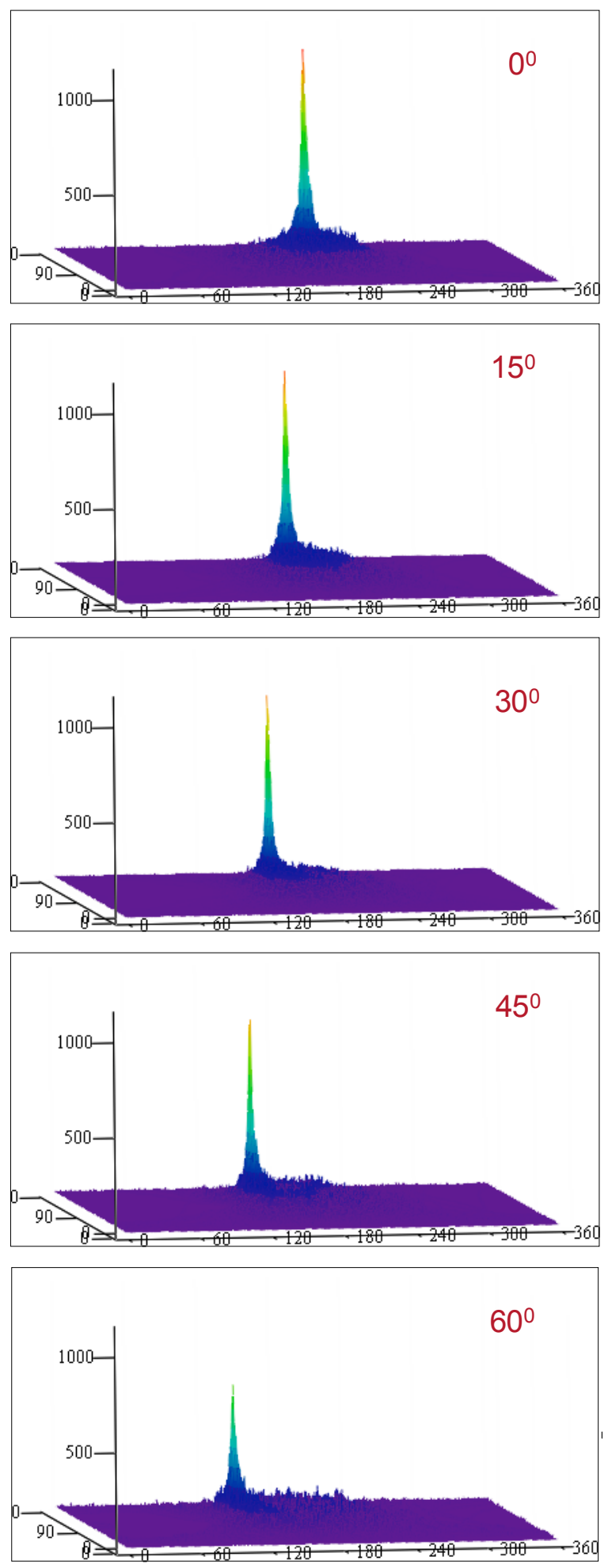

Fig. 2. $1408 \mathrm{keV}$ point spread function versus angle. The images are simple back-projection; no image reconstruction was done. The $\mathrm{x}$-axis runs from $0^{\circ}$ to $360^{\circ}$ around the detector in the horizontal plane. The $y$-axis is $+/-$ 90 degrees in the vertical plane. The $\mathrm{z}$ axis represents the number of events projecting to that pixel in space. 


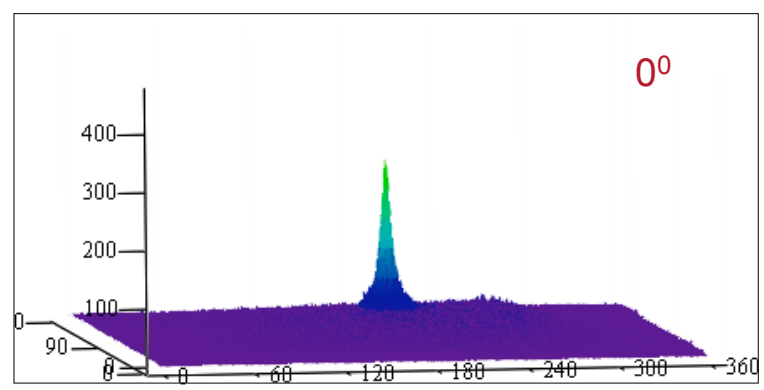

image 1
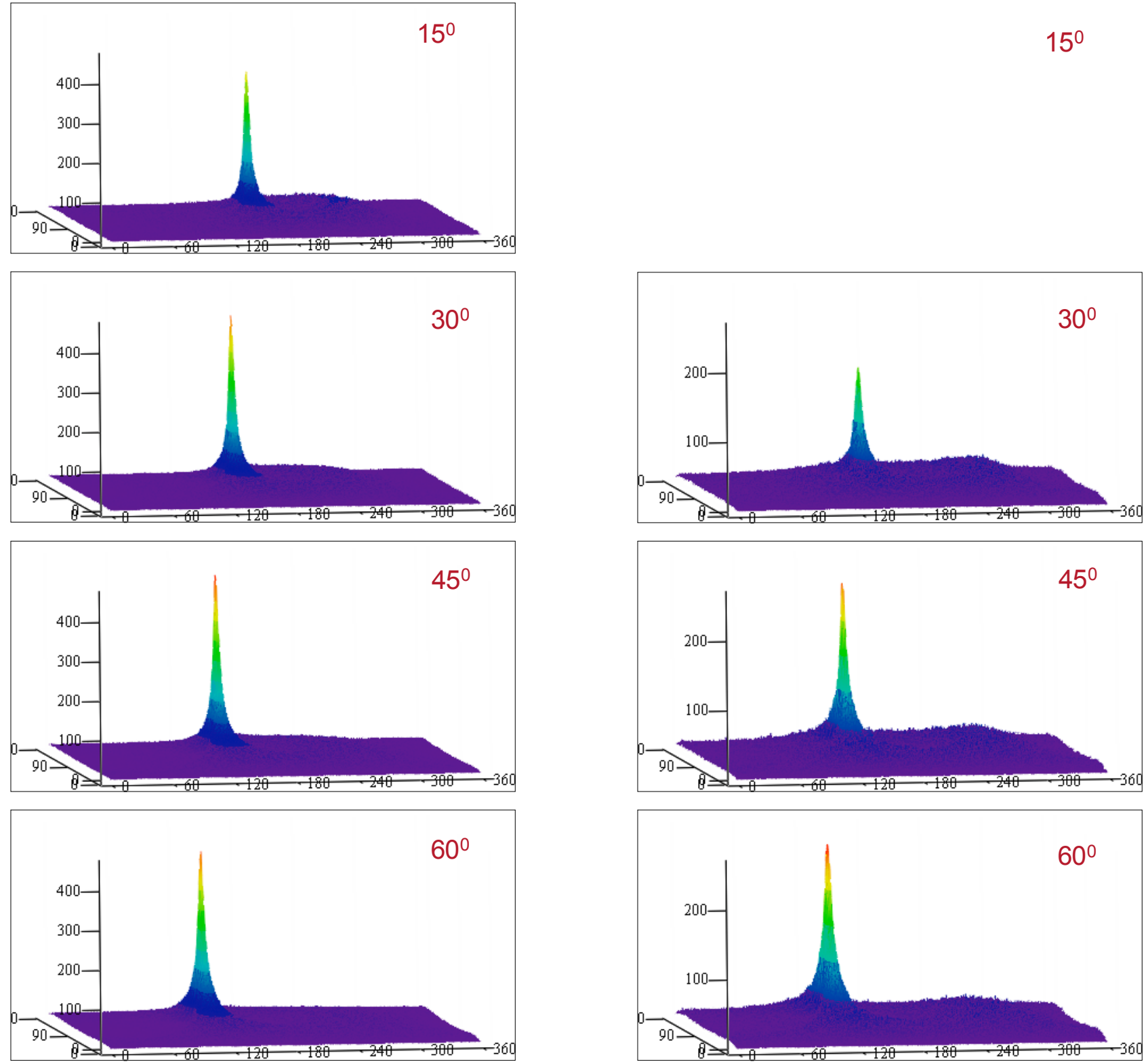

Fig. 3. 344 point spread function versus angle. The efficiency begins to drop at low angles.

Fig. 4. $244 \mathrm{keV}$ point spread function versus angle. The imaging fails at low angle scattering (near zero degrees) due to the small amount of energy deposited in the first interaction. 
Figures 2-4 show point source images taken at $1408 \mathrm{keV}$, $344 \mathrm{keV}$ and $244 \mathrm{keV}$ respectively. These images represent the PSF as a function of angle, where the angle is taken with respect to the detector axis (i.e. $0^{\circ}$ is directly in front of the system). Note that the imaging efficiency at low scatter angles (near $0^{\circ}$ ) degrades at lower energies. Also, the PSF becomes asymmetric for all energies at higher scatter angles. Both these effects are due to the geometrical bias of the detector and are described in detail in section VII.

\section{Angular Resolution Metric (ARM)}

The resolution is defined as the full-width half-max (FWHM) of the distribution of the angular resolution metric (ARM). The ARM is found by plotting the difference between the geometric angle (based on the measured positions of interactions and known source location) and the scatter angle predicted by the Compton scatter formula. Fig. 5 shows the ARM distribution for a $244 \mathrm{keV}$ and $1408 \mathrm{keV}$ point source. Note that the $244 \mathrm{keV}$ distribution is located on top of a smaller and broader secondary distribution. This second distribution comes from the Compton continuum events underneath the $244 \mathrm{keV}$ photopeak, as seen in the energy spectrum (Fig. 1). This background was subtracted for calculating the resolution of the $244 \mathrm{keV}$ events.
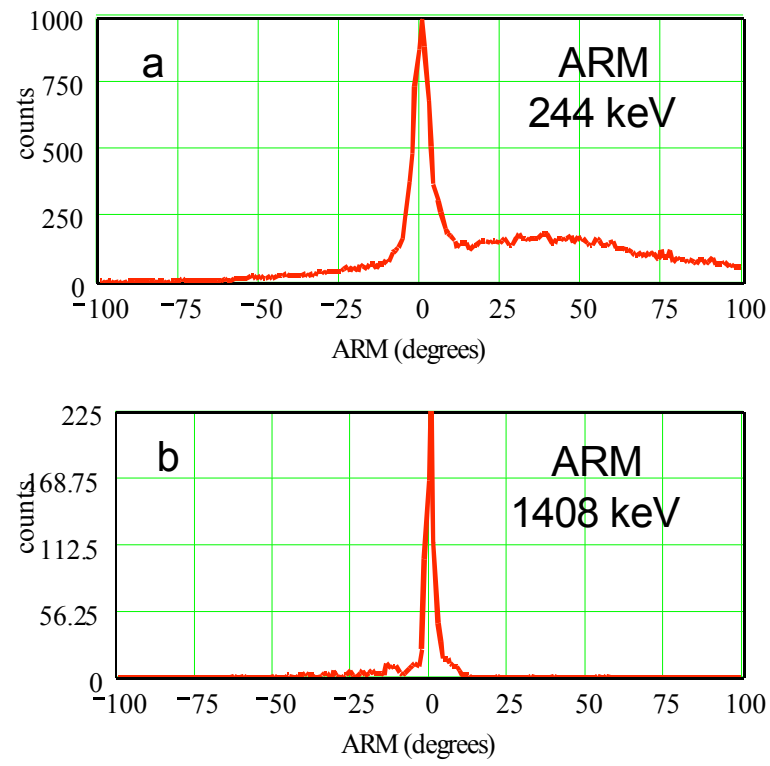

Fig. 5. Angular Resolution Metric for a $244 \mathrm{keV}$ and $1408 \mathrm{keV}$ point source. The resolution is defined as the FWHM of the ARM distribution.

\section{RESULTS}

The resolution for a range of energies and angles was determined by making an ARM distribution for each data set. Fig. 6 shows the FWHM resolution as a function of angle for the Monte Carlo simulation at $344 \mathrm{keV}$. The main components that contribute to the resolution (Doppler broadening, energy and position uncertainty) were quantified separately. At this energy, all three effects are significant. This is in contrast with the results at $1408 \mathrm{keV}$ (Fig. 7). Here, the Doppler broadening is a small effect and the energy uncertainty is negligible. Instead, the position uncertainty is the dominant contributor.

Figure 7 also shows the measured resolution as a function of angle from $0^{\circ}$ to $60^{\circ}$. The measured resolution is seen to be better than the simulated resolution at $45^{\circ}$. However, this measurement had few events in the photopeak making the statistical uncertainty high.

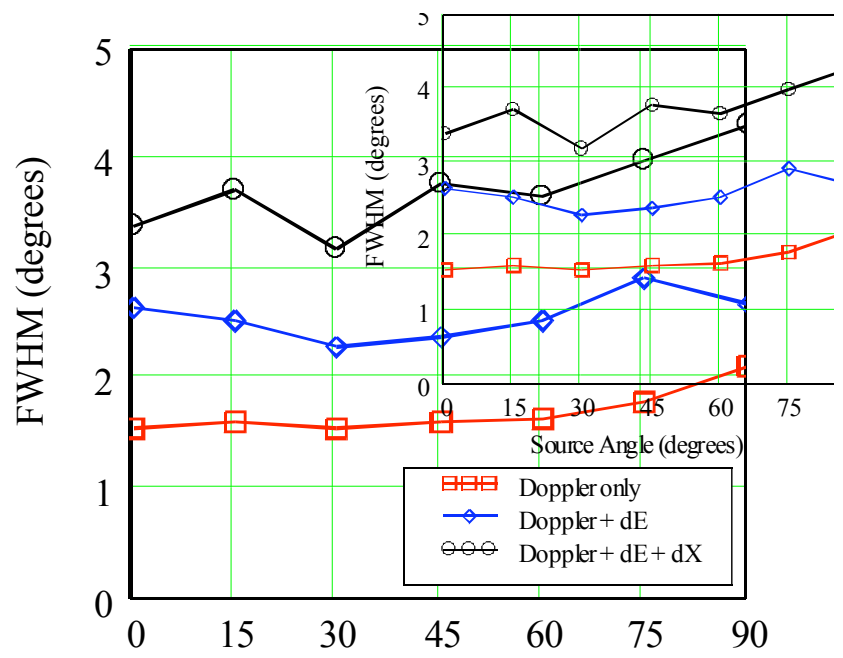

Fig. 6. Simulated resolution at $344 \mathrm{keV}$. The resolution as a function of source angle (relative to the detector axis) is shown for the case of Doppler only, Doppler plus energy uncertainty, and Doppler plus energy and position uncertainty.

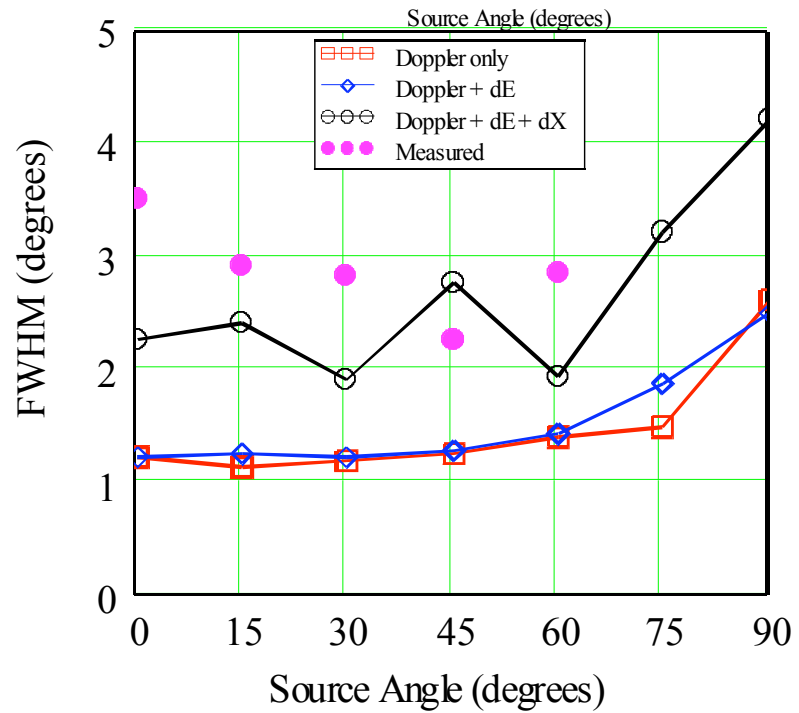

Fig. 7. Resolution at $1408 \mathrm{keV}$. The simulated and measured resolution for this system in shown. The simulated resolution has been broken down into constituent components. Note that the energy uncertainty $(<2 \mathrm{keV}$ per interaction) is negligible at this energy.

Fig. 8 shows the measured and simulated resolution as a function of energy. For these measurements, all source were placed at $30^{\circ}$ relative to the detector axis. 


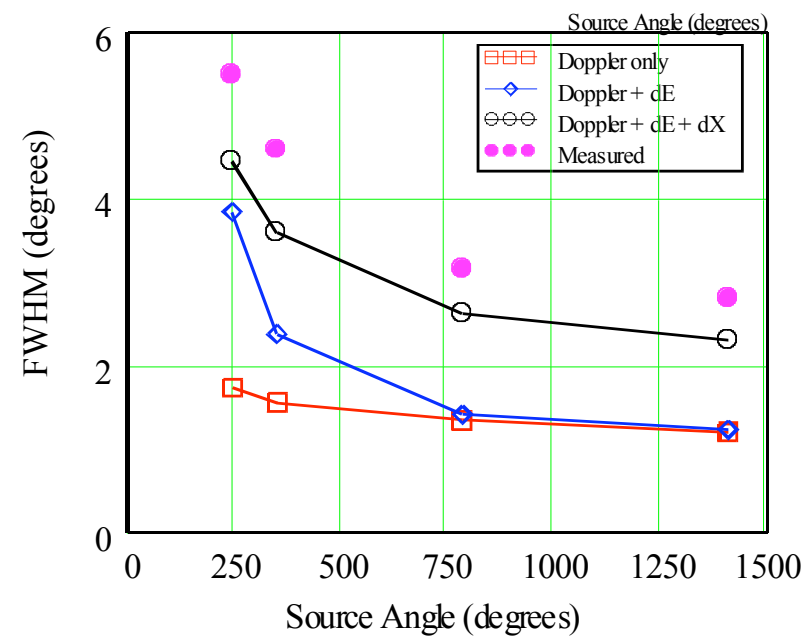

Fig. 8. Resolution versus energy. All sources were at $30^{\circ}$ relative to the detector axis.

\section{GEOMETRICAL BIAS}

It was shown that the resolution changes depending on the energy and location of the source. In addition, the shape of the PSF also change. The PSF changes from symmetric to asymmetric for sources at large angles with respect to the detector axis. This effect is shown in Fig. 9a, part a and can be explained as follows: following a Compton interaction in the first detector, a $\gamma$-ray has an equal chance of scattering left or right (due to symmetry). However, if the $\gamma$-ray came in from a large angle then scattering to the right (relative to the figure) will cause it to enter the system while scattering to the left will cause it to escape. This results in a biased sampling of scatter angles and a predominance of events that project to one side of the source (Fig. 10).

Another bias due to geometry is seen for low energy events. Low energy events (below $300 \mathrm{keV}$ in our prototype system) do not image well at shallow scatter angles because the initial energy deposited is too small. This energy is either in the noise or below the minimum energy threshold for the detector (Fig. 9b).

Knowing the shape of the point spread function is important for proper image reconstruction. These factors also affect the field of view and efficiency of the system and thus the sensitivity.
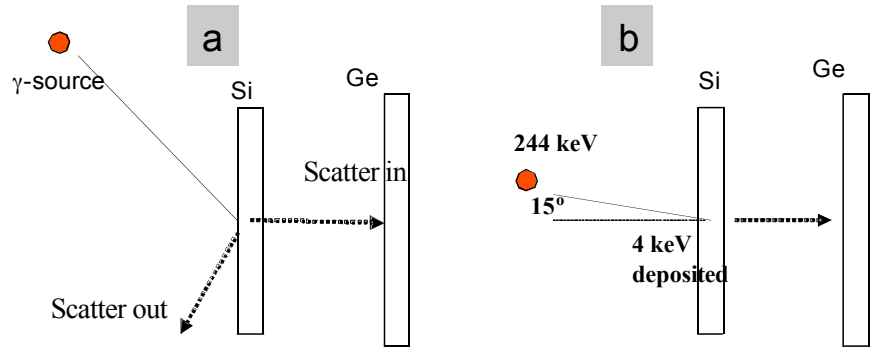

Fig. 9. Detector geometry (size, thickness and spacing between detectors) affects that scatter angles that can be accepted. This biasing of scatter angles perturbs the point spread function.

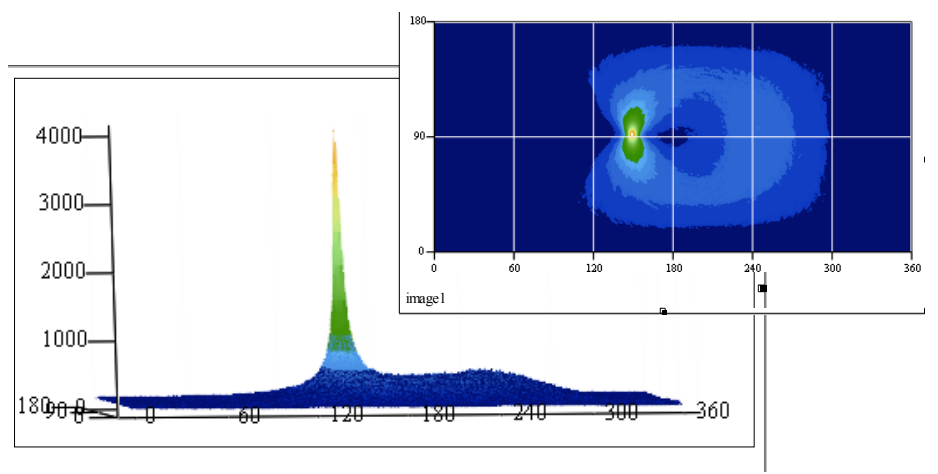

Fig. 10. Image of a $344 \mathrm{keV}$ point source at $30^{\circ}$ with respect to the detector axis. The PSF is asymmetric due to bias from the detector geometry only accepting certain scatter angles. The image is also shown in 2-D projection.

\section{DISCUSSION}

The measured resolution averaged from $3^{\circ}$ to $4^{\circ}$ across most of the energy range and field of view of this instrument. While this result was quite satisfactory, it tended to be $\sim 0.5^{\circ}$ higher than predicted by simulations, even after accounting for energy and position uncertainty as well as Doppler broadening. This suggests systematic errors in the system that were unaccounted for.

One likely possibility for this error is the precision with which the detector alignment is known. A scanning measurement with a fine beam collimator was used to help determine the relative positions of the silicon and germanium crystals. In addition, a trial and error optimization was done in software by moving the relative positions to obtain the best resolution. A combination of these two lead to an estimated alignment uncertainty of $\sim 0.5 \mathrm{~mm}$ in $\mathrm{x}, \mathrm{y}$ and $\mathrm{z}$ which is sufficient to explain the $0.5^{\circ}$ resolution broadening.

The field of view is limited to about $+/-60^{\circ}$ for high energy events, where the efficiency begins to fall. At low energy, the field of view is limited not at high angles but rather at low angles where shallow scattering results in insufficient energy deposition.

All these issues are being addressed with the next generation system. It will have two layers of silicon plus two layers of germanium detectors resulting in a greater stopping power for high energy photons. The spacing will also be optimized to allow for a greater acceptance of scatter angles. This will improve the imaging in the low energy range (below $200 \mathrm{keV}$ ) and will give a more symmetric PSF.

\section{REFERENCES}

[1] V. Schonfelder, "Lessons Learnt from COMPTEL for Future Telescopes", New Astronomy Reviews, Feb 2004 (48) pp. 193-198.

[2] M. Cunningham et. al., "First-Generation Hybrid Compact Compton Imager" IEEE Nuclear Science Conference Proceedings, Puerto Rico, 2005. 